OLYMPIC BASICS - Geschichtliche, bildungstheoretische und sozialwissenschaftliche Grundlagen 


\section{Die antiken Olympischen Spiele}

Modell oder Mythos?

Manfred Lämmer

In seiner viel beachteten Rundfunkrede, die am 4. August 1935 weltweit ausgestrahlt wurde, erklärte der Gründer der Olympischen Bewegung, Pierre de Coubertin: „Die Olympischen Spiele feiern heißt sich auf die Geschichte berufen" (Coubertin, 1966, S. 154). Der französische Baron stand, wie zahlreiche seiner Zeitgenossen, während seines ganzen Lebens unter dem bestimmenden Einfluss neuhumanistischen Gedankenguts des 19. Jahrhunderts und war fest davon überzeugt, die Griechen hätten sozusagen in einem einmaligen Schöpfungsakt das Prinzip des Wettkampfes erfunden, seinen Sinn definiert und einen Kanon ewig gültiger, unveränderlicher Werte hinterlassen. Heute wissen wir, dass das idealisierende und romantisierende Bild, dass sich die Vertreter von Turnen und Sport im Allgemeinen und die Ideologen des Olympismus im Besonderen damals von der antiken Athletik und den Spielen im alten Olympia machten, nicht der Realität entsprach. Die olympischen Funktionäre projizierten noch lange ihre (gut gemeinten) Ziele, Vorstellungen und Forderungen in die Antike, um ihnen mit Hilfe eines anerkannten Kulturerbes mehr Gewicht zu verschaffen. Sie sahen das alte Griechenland nicht, wie es Historikern und Archäologen aus den Originalquellen entgegentrat, sondern so, wie man es zur Legitimation eigener Ziele benötigte (Weeber, 1991, S. 7-13). Diese Strömung gipfelte in der Vorlage des sog. Hellas-Planes, dem Anfang der 1980er Jahre nach dem Boykott der Olympischen Spiele in Moskau und unter dem Eindruck der massiven Bedrohung der Olympischen Bewegung durch die Politik insgesamt vom Hellenischen Olympischen Komitee und der griechischen Regierung gemeinsam gemachten Vorschlag, die Olympischen Spiele in Zukunft an ein und demselben Ort in Griechenland zu veranstalten, um durch die Rückkehr an ihren geografischen und geistigen Ursprung, von der man sich gewissermaßen eine moralische Selbstreinigung erhoffte, die ursprüngliche Olympische Idee in ihrer unverfälschten Form wiederzugewinnen, die Spiele dem Würgegriff von Politik und Kommerz zu entreißen und vor dem moralischen Niedergang zu bewahren. Auch die Vertreter der Bewerbung Athens um die Austragung der Olympischen Spiele 1996 und 2004 
führten diese Argumente wieder ins Feld. Doch der Plan wurde bereits im Vorfeld des Olympischen Kongresses 1981 in Baden-Baden in einer Abstimmung unter den Nationalen Olympischen Komitees einhellig abgelehnt. Diese an irrationale Gefühle appellierende Idee ist inzwischen vom Tisch, weil sie den Forschungsergebnissen der kritischen Altertumswissenschaft in keiner Weise standhielt (Lämmer, 1980; Fricke, 1982).

\section{Die griechische Athletik und die gesellschaftliche Elite}

Die athletischen Wettkämpfe der Griechen entstanden nicht auf dem Reißbrett eines Pädagogen, sondern entwickelten sich unter konkreten politischen und sozialen Gegebenheiten zwischen dem 8. und 6. Jahrhundert v. Chr. In der damaligen Feudalordnung, deren Grundstrukturen bis in die mykenische Epoche zurückverfolgt werden können, befand sich die gesamte politische und wirtschaftliche Macht in den Händen aristokratischer Großgrundbesitzer, die über eine Bevölkerung von Freien, Halbfreien und Sklaven herrschten und ihre Stellung durch militärische Macht sicherten. Zentrale Forderung ihrer Standesethik war das Streben nach Ruhm und Ehre. Dieses Bedürfnis beruhte darauf, dass der Grieche der frühen Zeit eine zutiefst pessimistische Lebensanschauung hatte: Er glaubte - wie die Epen Homers eindrucksvoll zeigen - nicht an ein Weiterleben nach dem Tode - zumindest nicht in einer lohnenswert erscheinenden Form. Daher sah er den Sinn seiner Existenz darin, in der kurzen Zeitspanne, die ihm zur Verfügung stand, durch großartige Leistungen und Erfolge dem befürchteten Vergessen durch spätere Generationen zu entrinnen.

Diese Handlungsmaxime fasste einer der Helden des Trojanischen Krieges in den Vers, der seit mehr als 100 Jahren in allen einschlägigen Standardwerken zur Geschichte der griechischen Athletik zitiert wird: „Immer der beste [sic] sein und überlegen den anderen" (Homer, Ilias VI, 208).

Natürlich ist dieses Zitat aus Homers Werk sicherlich manchmal überstrapaziert worden, aber im Grunde gibt es das Lebensgefühl der frühgriechischen Aristokratie, das wir ja aus vielen anderen Passagen in Ilias und Odyssee erschließen können, adäquat wieder (Weiler, 1975). Bewährungsfeld des Adligen war der Agon. Darunter verstand man ursprünglich die Versammlung der wehrfähigen Männer. Später bezeichnete der Begriff die wesentliche Aktivität dieses Kreises, nämlich Krieg und Schlacht auf der einen und den athletischen Wettkampf auf der anderen Seite. Ob wir es wahrhaben wollen oder nicht: Die griechische Athletik 
hat sich in militärischem Kontext entwickelt und blieb Jahrhunderte lang auf ihn bezogen (Lämmer, 1985). Der Phäakenkönig Alkinoos drückt es in Homers Odyssee so aus: „Es gibt keinen größeren Ruhm für einen Mann als den, den er mit seinen Händen und Füßen erringt" (Homer, Odyssee VIII, 147-148).

Abbildung 1: Der Waffenlauf-ein Zeichen für den kriegerischen Ursprung der griechischen Athletik

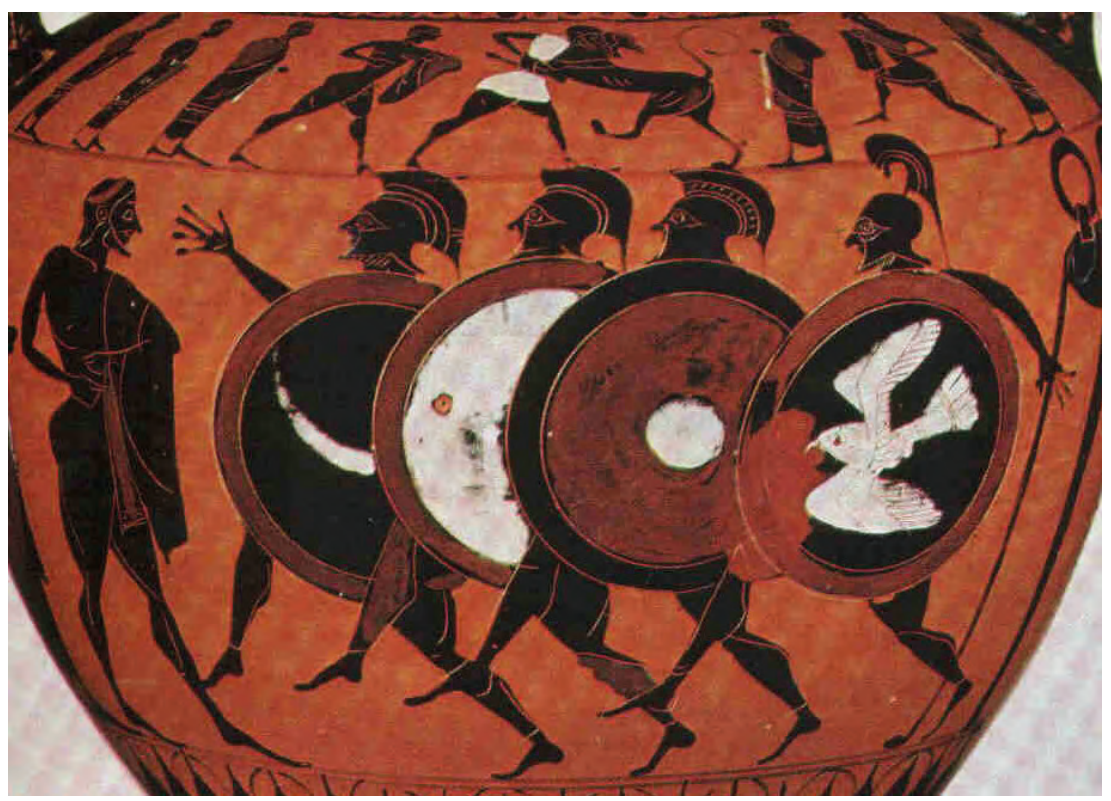

(Matthias Kabel, CC-BY-SA 2.5, via Wikimedia Commons-Zuschnitt)

Dieser Ausspruch zeigt die überraschend geringe Wertschätzung geistiger und künstlerischer Leistungen in der frühen Phase griechischer Geschichte. Philosophen, Dichter und Künstler standen eindeutig im Schatten derer, die Schlachten schlugen oder den Gegner im sportlichen Wettkampf besiegten.

Obwohl es in der europäischen Frühe eine ganze Reihe vergleichbarer Gesellschaftsordnungen gab, hat sich hinsichtlich der dauerhaften Etablierung athletischer Wettkämpfe nirgendwo eine ähnliche Entwicklung vollzogen. Eine schlüssige Erklärung dafür ist die Forschung bisher schuldig geblieben. Vielleicht liegt sie in einer spezifischen Situation, die im 6. Jahrhundert v. Chr. in Griechenland entstand. 
$\mathrm{Zu}$ dieser Zeit geht die politische Macht vom Adel auf eine breitere Schicht von Kaufleuten und Manufakturbesitzern über, die nach Einführung der Geldwirtschaft und durch die einsetzende Kolonisation zu Wohlstand und Einfluss gekommen war. Es entsteht die Polis (Stadtstaat), jenes unverwechselbare griechische Gemeinwesen, das durch den freien Willen gleichberechtigter Bürger gestaltet wird. Doch da an der Spitze dieses Bürgertums vielfach Volksführer aus Kreisen des alten Adels stehen, entwickelt es keine eigenen neuen Wertvorstellungen, sondern übernimmt die des Adels.

So blieb die aristokratische Standesethik im Kern bestimmend für das Bürgertum der klassischen und sogar der hellenistischen Zeit. Dabei spielten die Werke Homers eine wichtige Rolle, denn mit ihrer Hilfe lernte der junge Grieche nicht nur Lesen, Schreiben, Geografie, Geschichte und Religion, die Helden der homerischen Epen verkörperten auch die Erziehungsprinzipien und Lebensideale, die für die Polis-Jugend gültig waren.

Und so entstanden als Ausdruck dieser agonalen Orientierung in ganz Griechenland hunderte periodischer Kultfeste mit athletischen, hippischen und musischen Wettkämpfen, insbesondere die Panhellenischen Spiele. Erfolge im Wettkampf bestimmten auch weiterhin das Ansehen eines Bürgers in seiner Stadt wie vorher das eines adligen Kriegers unter Seinesgleichen. Soziale Mobilität für Angehörige unterer und mittlerer Schichten aufgrund athletischer Erfolge dürften in klassischer Zeit Ausnahmen gewesen sein. In einer Reihe von Fällen, die den gegenteiligen Eindruck erwecken, stellt sich bei näherer Betrachtung heraus, dass die Zugehörigkeit zu einer arrivierten Schicht nicht die Folge athletischer Leistungen, sondern vielmehr deren Voraussetzung gewesen ist.

\section{Die antiken Olympischen Spiele}

Die Olympischen Spiele, die wir heute erleben, sind keine Kopie eines antiken Vorbildes. Die folgenden Ausführungen sollen als Orientierungshilfe dienen, um die Spannung zwischen dem olympischen Geschehen unserer Tage und der antiken Wirklichkeit besser zu verstehen. Dabei werden sich sowohl Parallelen als auch überraschende Gegensätze auftun. 
Abbildung 2: Modell des antiken Olympia mit seinen Kult-und Sportstätten

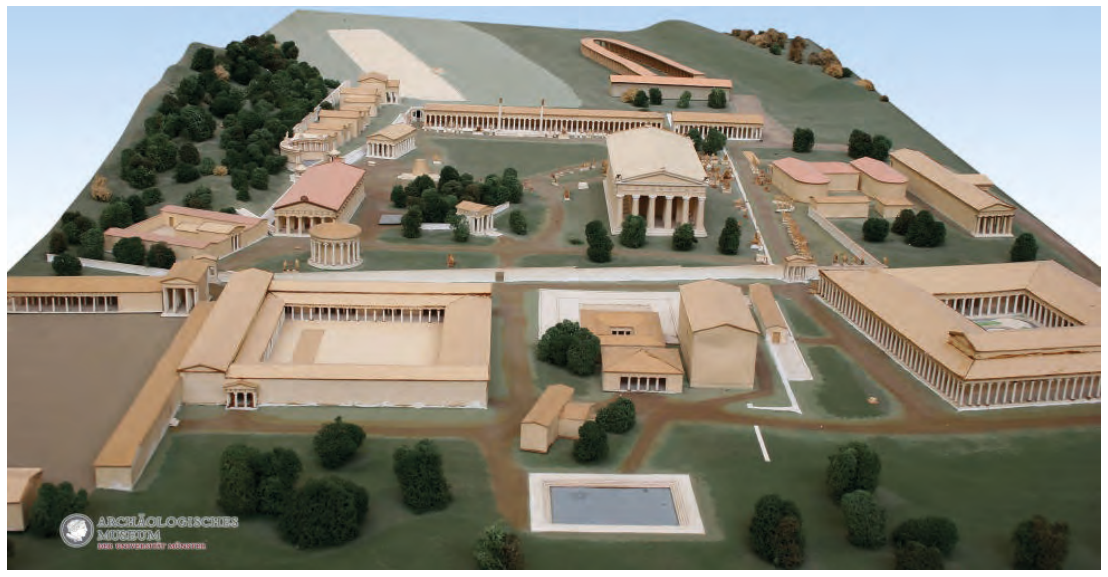

(Archäologisches Museum der WWU Münster, Inv. AM 1, Foto Robert Dylka)

Die antiken Olympien fanden der Überlieferung nach von 776 v. Chr. bis $393 \mathrm{n}$. Chr. alle vier Jahre jeweils im Juli/August in Olympia, dem berühmtesten Kultort der griechischen Welt, im nordwestlichen Winkel der Halbinsel Peloponnes zu Ehren des höchsten griechischen Gottes Zeus statt. Im Gegensatz zu heute wurden sie stets am gleichen Ort und durch den gleichen Veranstalter, die nahegelegene Stadt Elis, ausgerichtet. Es gab also keine Bewerbung um die Austragung, wie wir sie heute erleben. Es war Pierre de Coubertin, der das Prinzip der Wanderspiele als ein zentrales Element seiner olympischen Philosophie einführte.

Die sechstägige Veranstaltung wies folgende Wettbewerbe auf:

Athletische Agone

- Stadionlauf

- Doppellauf

- Langlauf

- Fünfkampf (Pentathlon)

- Waffenlauf
$192 \mathrm{~m} \quad$ ab 776 v.Chr.

$384 \mathrm{~m}$ ab $724 \mathrm{v} . \mathrm{Chr}$.

$3.840 \mathrm{~m}$ ab 720 v.Chr.

ab 708 v.Chr.

$384 \mathrm{~m}$ ab $520 \mathrm{v} . \mathrm{Chr}$. 
Kampfsport

- Ringen

ab 708 v.Chr.

- Boxen

ab 688 v.Chr.

- Pankration

ab 648 v.Chr.

Hippische Agone

- Wagenrennen

ab 680 v.Chr.

- Reitwettbewerbe

ab 648 v.Chr.

Die athletischen Disziplinen wurden im Stadion ausgetragen, die Pferdewettbewerbe im Hippodrom. Entgegen immer wieder zu hörenden Behauptungen gab es in Olympia kein Theater und keine musischen Darbietungen, wie an vielen anderen Kultorten, auch wenn sich Coubertin wiederholt auf diese berief und olympische Kunstwettbewerbe einführte, bei denen die Teilnehmer und Teilnehmerinnen von 1912 bis 1948 Medaillen erringen konnten. Zu den Wettkämpfen in Olympia strömten Tausende von Zuschauern aus dem gesamten griechischen Kulturbereich. 
Abbildung 3: Der tunnelartige Zugang zum Stadion von Olympia

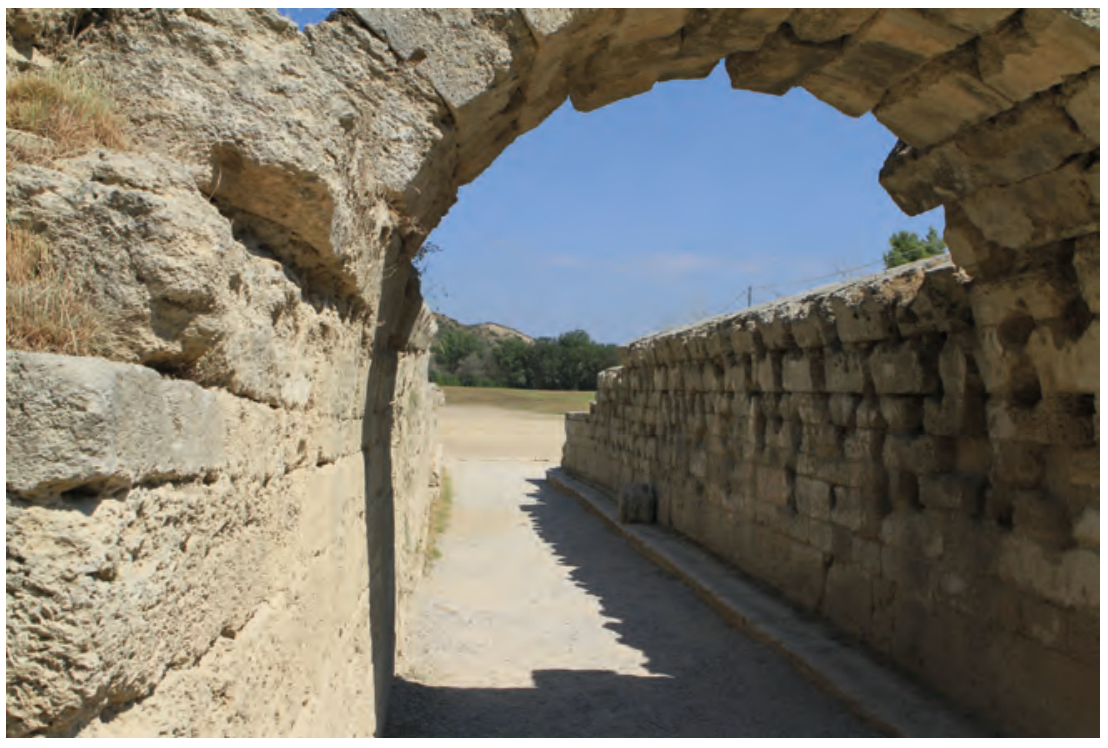

(picture alliance/ANE)

Das Stadion fasste 40.000 Zuschauer, die auf grasbewachsenen Erdwällen Platz nahmen. Während heute die ganze Welt zum Stadion wird, weil Milliarden von Menschen das Geschehen über das Fernsehen oder digitale Medien verfolgen können, mussten die Schlachtenbummler in der Antike, wie natürlich auch die Athleten, oft wochenlange, anstrengende und kostenträchtige Reisen auf sich nehmen, um vor Ort dabei zu sein. So rühmte sich ein Bäcker aus Mazedonien auf seinem Grabstein, zwölfmal zu den Olympischen Spielen gereist zu sein (Ebert, 1980, S. 89).

Das Wettkampfprogramm war also sehr überschaubar. Eine Beschreibung der einzelnen Sportarten und Disziplinen ist in diesem Beitrag nicht beabsichtigt. Dazu liegen zahlreiche Standardwerke vor (Ebert, 1980; Swaddling, 2004; Sinn, 2004; Decker, 2012). Im Hinblick auf die Zielsetzung dieses Bandes geht es im Folgenden vielmehr um eine Darstellung der Wesenszüge und des Sinngehalts der antiken Olympischen Spiele und um einen Vergleich mit ihrem modernen Pendant. 
Das Fest des Zeus

Um Wesen und Bedeutung der antiken Olympischen Spiele zu verstehen, muss man sich vor allem bewusst machen, dass sie keine selbständige Sportveranstaltung waren, sondern Teil eines religiösen Festes zu Ehren des höchsten griechischen Gottes Zeus. Diese enge und unauflösliche kultische Bindung bewirkte den Aufstieg der Spiele und ihre Anerkennung durch alle Griechen, sicherte jahrhundertelang ihre Stellung, führte aber im Zuge der Christianisierung des Römischen Reiches auch zu ihrem Ende. Die modernen Olympischen Spiele sind dagegen ein profanes Sportfest. Der Versuch Pierre de Coubertins, die verlorene religiöse Sinnmitte durch das Postulat einer religio atbletae (Coubertin, 1966, 150) wenigstens teilweise zurückzugewinnen, blieb diffus und ohne nachhaltige Wirkung. Dagegen gelang es ihm und seinen Nachfolgern, durch das olympische Zeremoniell und die olympischen Symbole Athleten und Zuschauer feierlich einzustimmen und das Geschehen emotional zu erhöhen. 
Abbildung 4: Die Zeusstatue des Phidias, eines der sieben Weltwunder der Antike. Versuch einer Rekonstruktion

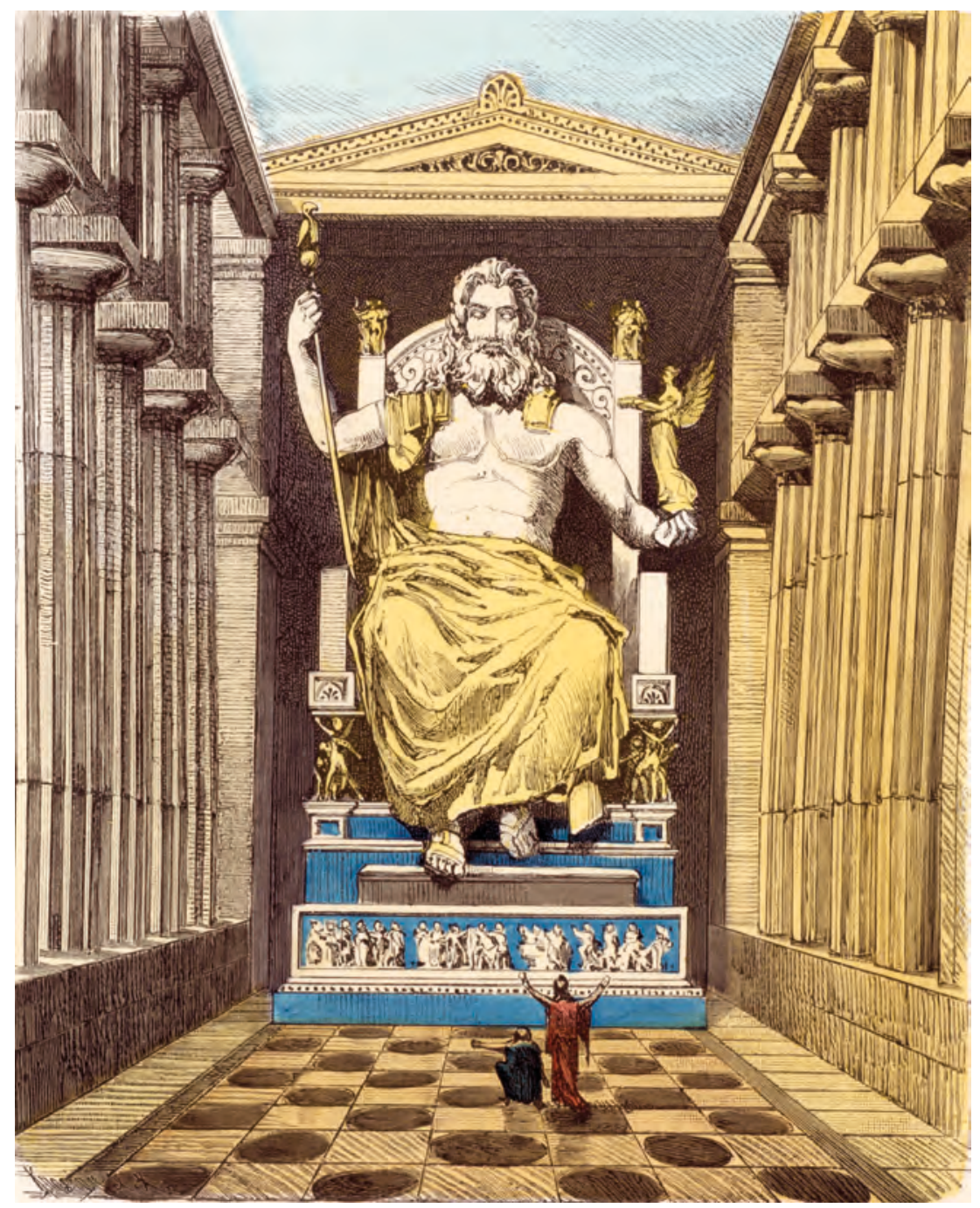

(picture-alliance/akg-images) 


\section{Nationalität und Internationalität}

Das wohl wichtigste sinnstiftende Wesensmerkmal der modernen Olympischen Spiele ist ihre Internationalität. Die Zahl der teilnehmenden Länder wurde geradezu zum Gradmesser ihres Ansehens. Getreu der Devise Coubertins „All Games - All Nations“ sollen Athleten aus möglichst allen Ländern der Welt ohne Diskriminierung auf Grund ihrer Rasse, Religion oder politischen Anschauung an den Start gehen. Das IOC war einer verlockenden Universalität willen sogar mehrere Male in Gefahr, andere grundlegende Prinzipien zu opfern (Olympische Charta, 2014).

Die antiken Spiele hatten dagegen einen strikt nationalen Charakter: Es waren ausschließlich Griechen zugelassen. Bis zum Ende der klassischen Zeit galt nur der als Grieche, der von griechischen Eltern abstammte. Außerdem musste er das volle Bürgerrecht einer Polis besitzen. Diese Bestimmung schloss von vornherein 50 Prozent der männlichen Bevölkerung aus. Unter dem Eindruck der Eroberung des Vorderen Orients durch Alexander den Großen gegen Ende des 4. Jahrhunderts v. Chr. und der dadurch ausgelösten Hellenisierung führender Schichten in dieser Region änderte sich diese starre Auffassung. Ungeachtet seiner blutsmäßigen Herkunft wurde nun jeder, der in einem Gymnasium eine umfassende körperliche und geistige Bildung genossen hatte und sich zur griechischen Kultur und Religion bekannte, als Grieche angesehen und zu den Olympischen Spielen zugelassen. Formal wurde der nationale Charakter der Olympischen sowie der anderen Panhellenischen Spiele (Pythien, Nemeen und Isthmien) auch in späterer Zeit nicht aufgegeben, obwohl die ethnische und soziale Herkunft eines Wettkämpfers kaum noch überprüft worden zu sein scheint.

In klassischer Zeit kamen die Athleten und Zuschauer zwar aus verschiedenen, unabhängigen Stadtstaaten, die häufig miteinander rivalisierten oder sogar gegeneinander Krieg führten, doch feierten sie das Fest in Olympia als Ausdruck einer bestehenden Gemeinschaft mit gleicher Sprache und Religion, gleichen Sitten und gesellschaftlichen Grundwerten. Über das unmittelbare festliche und sportliche Erlebnis hinaus sollten die Spiele aber nichts bewirken. Sie dienten weder der politischen Einigung aller Griechen noch der Verständigung mit den Nichtgriechen, die man als Barbaren bezeichnete und von den Spielen ausschloss, um sich bewusst von ihnen abzugrenzen. Wenn die Griechen in Olympia Ansätze eines politischen Zusammengehörigkeitsgefühls zeigten, geschah dies nur unter dem Zwang gemeinsamer Bedrohung von außen und richtete sich immer gegen jemanden. 
Aus historischer Sicht haben diejenigen nicht Unrecht, die die Spiele im antiken Olympia mit den Deutschen Turnfesten, den slawischen Sokol-Festen oder den jüdischen Makkabiaden verglichen haben. Die internationale Ausrichtung der modernen Olympischen Spiele ist eine ureigene Schöpfung Pierre de Coubertins und entspringt den spezifischen Absichten, die er mit ihnen verband. Um die Internationalität zu betonen, hielt er auch am Prinzip der Austragung der Spiele an wechselnden Orten fest.

\section{Olympia im Dienste des Friedens?}

Die moderne Olympische Bewegung erhebt den Anspruch, einen spezifischen Beitrag zur Völkerverständigung und zur Förderung des Weltfriedens zu leisten. Die Frage, auf welche konkrete Weise diese begrüßenswerten Ziele erreicht werden sollen, blieb bisher weitgehend unbeantwortet. Stattdessen wird vielfach das angeblich antike Vorbild beschworen: Bereits bei den alten Hellenen hätten die Olympischen Spiele im Dienste des Friedens gestanden. Wenn die Boten aus Elis den Beginn der heiligen Festzeit verkündet hätten, habe man in ganz Griechenland die Waffen niedergelegt. In Wahrheit haben die Griechen niemals von einem olympischen Frieden gesprochen, sondern von der Ekecheiria, einem Begriff, den man wohl am treffendsten mit Immunität wiedergibt (Lämmer, 1982; Höfer, 1994). Sie beruhte auf einer Vereinbarung der Eleer mit ihren unmittelbaren Nachbarn aus dem 8. Jahrhundert v. Chr., der in der Folgezeit alle griechischen Stadtstaaten beitraten, und bestand im Wesentlichen aus drei Bestimmungen (Lämmer, 1982):

- Das Heiligtum von Olympia und das Territorium des Staates Elis wurden für neutral und unverletzlich erklärt.

- Während eines Zeitraums von drei Monaten im Sommer eines Olympien-Jahres standen alle Wettkämpfer, Funktionäre und Zuschauer samt ihrem Eigentum unter göttlichem Schutz. Sie konnten ungehindert nach Olympia reisen und sich wieder nach Hause zurückbegeben, selbst wenn ihr Weg durch das Gebiet eines Staates führte, der sich mit der eigenen Polis gerade im Kriegszustand befand.

- Alle Staaten, die der olympischen Kultgemeinschaft angehörten, verpflichteten sich zu Sanktionen gegen jeden, der die Bestimmungen der Ekecheiria verletzte. Derartige Strafmaßnahmen trafen sogar die Großmächte Sparta und Athen.

Das heute immer noch weitverbreitete ideologische Dogma einer totalen und für ganz Griechenland verbindlichen Waffenruhe für die Dauer 
der Olympischen Spiele ist pure Fiktion. Die Olympic Truce bedeutete kein Ende aller Kriege, sondern sicherte die Veranstaltung der Spiele trotz der Kriege. Während die modernen Olympischen Spiele in ihrer 100-jährigen Geschichte bereits dreimal Kriegen zum Opfer fielen, wurden die antiken Spiele länger als 1100 Jahre ohne eine einzige Unterbrechung gefeiert. Sie waren jedoch mit keiner friedensstiftenden Idee oder Programmatik verbunden. Der Gedanke, internationale sportliche Wettkämpfe planmäßig in den Dienst der Völkerverständigung und des Friedens zu stellen, geht vielmehr ebenfalls auf Pierre de Coubertin zurück.

Abbildung 5: Das Rennen mit dem Viergespann - der Höhepunkt der Wettbewerbe im Pferdesport

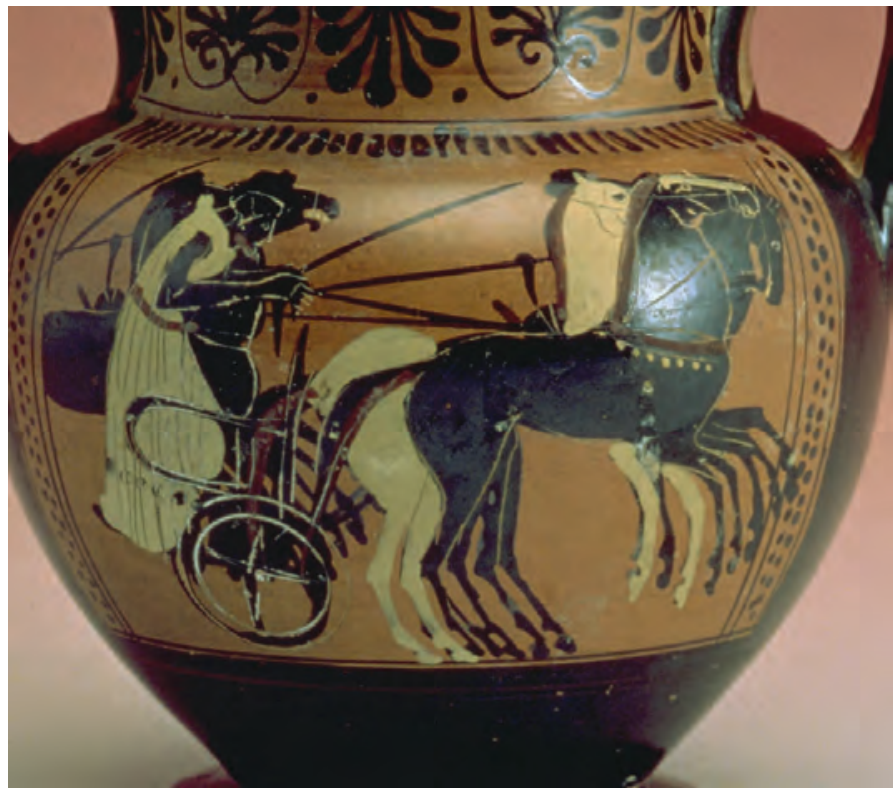

(IOC)

Frauen bei Olympischen Spielen?

Der Anspruch des weiblichen Geschlechts auf Gleichberechtigung im Sport ist heute in fast allen Ländern der Welt trotz der Verschiedenheit nationaler Traditionen und gesellschaftlicher Ordnungen im 
Prinzip anerkannt, obwohl noch nicht überall in wünschenswertem Maße verwirklicht. Die wachsende Beteiligung der Frauen am Spitzensport und ihre rapide steigenden Leistungen gehören zu den faszinierendsten Erscheinungen im Sport unserer Tage. Vor allem die Olympischen Spiele haben dadurch eine neue attraktive Dimension erhalten.

Die Verfechter des Frauensports haben zu dessen Propagierung vor allem gesundheitliche, pädagogische und emanzipatorische Argumente vorgebracht. Sie haben sich aber auch wiederholt auf die griechische Antike berufen. Um ihrer Behauptung mehr Glaubwürdigkeit zu verleihen, sammelten sie mit großem Fleiß alle überlieferten Fragmente und fügten sie zu dem idealen Gesamtbild zusammen, das man sich wünschte. Eine Überprüfung des Sachverhalts ohne ideologische Voreingenommenheit erbringt jedoch ein ernüchterndes Ergebnis: Abgesehen von einigen Besonderheiten im dorischen Stammesbereich waren die gesellschaftlichen Rollen der Geschlechter in Griechenland strikt festgelegt: Der Mann stand im öffentlichen Leben und war verantwortlich für Politik, Wirtschaft und Krieg, der Frau oblag dagegen ausschließlich die Sorge um Haus und Familie. Während der junge Mann freier Herkunft im Gymnasium die Bildung erhielt, die ihn dazu befähigte, seine Rechte und Pflichten als Vollbürger auszuüben, erhielt das Mädchen allenfalls Elementarunterricht im Lesen und Schreiben und wurde im Übrigen von der Mutter auf die praktischen Erfordernisse von Ehe und Haushalt vorbereitet. Hinzu kam ein weiterer wichtiger Umstand: Das Gymnasium entstand im 6. Jahrhundert v. Chr. als militärischer Übungsplatz der wehrfähigen Söhne der neu aufgestiegenen Bürgerklasse. Da aber die griechische Frau in der Politik und bei der Verteidigung der Stadt keine Rolle spielte, nahm sie weder an den Übungen im Gymnasium noch an öffentlichen Wettkämpfen teil. An dieser Beurteilung ändern auch einige aus der römischen Kaiserzeit überlieferte Kuriositäten nichts (Lämmer, 1981). Zu den Olympischen Spielen waren nur Männer zugelassen. Frauen hatten lediglich die Möglichkeit, Pferde oder Gespanne zu den hippischen Agonen zu schicken. Da nicht der bezahlte Jockey, sondern der Besitzer der Pferde als der eigentliche Wettkämpfer galt, kamen einige adlige Frauen so zu olympischem Ruhm, ohne dass dies von sportlicher Bedeutung war. Bei den immer wieder als Beweis vorgebrachten Läufen zu Ehren der Göttin Hera, die in Olympia stattfanden (aber nicht in zeitlichem Zusammenhang mit den Olympischen Spielen!), handelte es sich nicht etwa um sportliche Wettkämpfe, sondern um eine religiöse Zeremonie junger Mädchen aus Elis. Im Übrigen war es Frauen sogar unter Androhung der Todesstrafe untersagt, während der Festzeit den heiligen Bezirk von Olympia oder die Wettkampfstätten zu betreten. 
Abbildung 6: Vor den Ruinen des Hera-Tempels wird das Olympische Feuer der modernen Olympischen Spiele entzündet

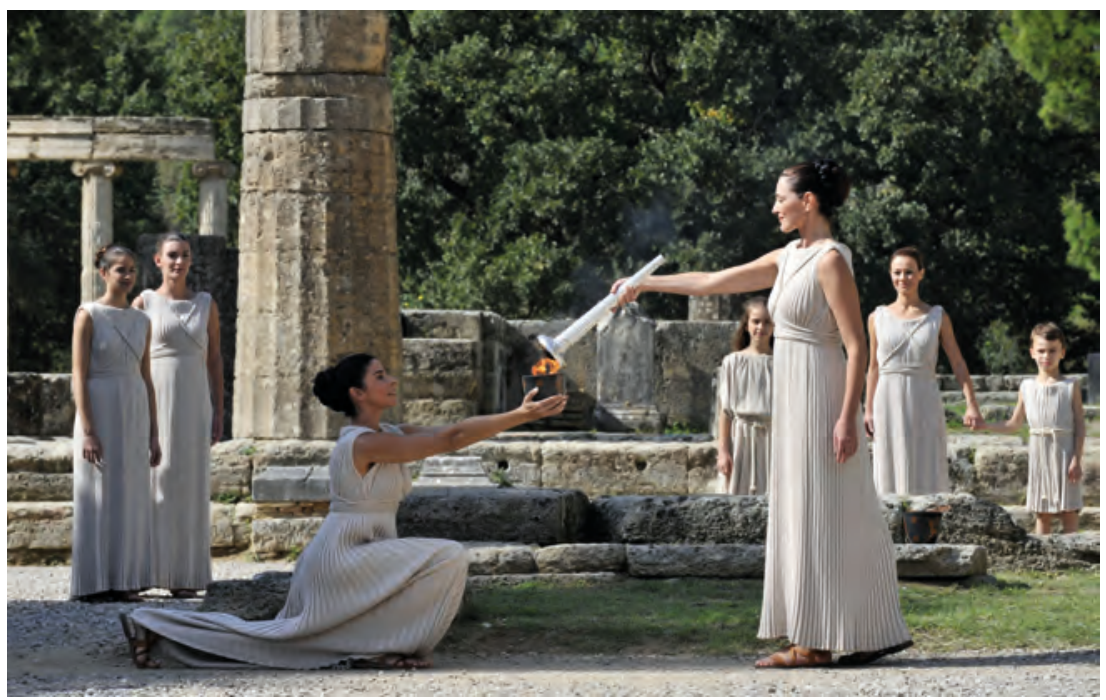

(2009/International Olympic Committee (IOC)/Juilliart, Richard)

Die Teilnahme der Frauen an den modernen Olympischen Spielen hat also keine antike Parallele. Sie ist Ergebnis der politischen, gesellschaftlichen und ökonomischen Emanzipation der Frau in den letzten 100 Jahren und musste gegen den erbitterten Widerstand führender Vertreter der Olympischen Bewegung, vor allem gegen Pierre de Coubertin, der sich dabei explizit auf die Antike berief, Schritt für Schritt durchgesetzt werden.

\section{Die Legende vom antiken Amateurideal}

Kein anderes Thema ist in der Geschichte der modernen Olympischen Spiele so lange und kontrovers diskutiert worden, wie das Verhältnis des Athleten zu materiellem Gewinn, ganz gleich, ob dies nun unter dem Stichwort Eligibility oder Kommerzialisierung geschah. Selbst heute gibt es noch olympische Funktionäre, die der festen Überzeugung sind, der tiefe moralische Sinn der Wettkämpfe im antiken Olympia - zumindest in klassischer Zeit - habe in der Verwirklichung eines Amateurideals gelegen. Sporthistoriker, die dieser Meinung sind, sollte es allerdings nicht mehr 
geben. Denn einschlägige Untersuchungen haben in diesem Punkte alle Illusionen zweifelsfrei beseitigt (Young, 1984).

Schon bei den ältesten uns überlieferten Wettkämpfen im 23. Gesang der Ilias des Homer stritten die adligen Krieger um wertvolle Preise. Auch in der so oft beschworenen klassischen Blütezeit der Olympischen Spiele im 5. Jahrhundert v. Chr. suchen wir nach einem Amateurideal vergebens. Zwar erhielten die Sieger bei den großen Panhellenischen Spielen auf dem Kampfplatz selbst nur einen Kranz. In ihren Heimatstädten wurden sie dagegen mit Belohnungen und Privilegien geradezu überschüttet. So zahlte die Stadt Athen bereits um 600 v. Chr. ihren Olympiasiegern Prämien von 500 Silberdrachmen, für die man damals immerhin einen Arbeiter drei Jahre entlohnen oder sich gar zehn Sklaven kaufen konnte. In fast allen griechischen Städten hatten Olympiasieger das Recht, ihr ganzes Leben lang im Rathaus (Prytaneion) zu speisen. Später ging man teilweise dazu über, den Gegenwert der Mahlzeiten - falls gewünscht - in Tagegeldern („Diäten“) auszuzahlen. In der römischen Kaiserzeit wurden die vorher von den autonomen Städten gewährten Privilegien durch einheitliche, kaiserlich garantierte Pensionen ersetzt. Aus den Zeugnissen geht klar hervor, dass wenigstens erfolgreiche Athleten, soweit sie nicht ohnehin aus einer begüterten Familie stammten, für die Zeit nach ihrer aktiven Karriere in hervorragender Weise abgesichert waren. Es muss ferner daran erinnert werden, dass nur wenige Wettkämpfe, darunter die vier großen Panhellenischen Spiele, sogenannte Kranzagone waren. Bei der Mehrzahl der regionalen und lokalen Veranstaltungen ging es dagegen um wertvolle Geld- und Sachpreise (Ebert, 1980, S. 78; Weeber, 1991, S. 113).

Es steht also unumstößlich fest, dass die Griechen keinen Unterschied zwischen Amateuren und Professionals gemacht haben. In welchen sozialen Verhältnissen jemand lebte und unter welchen Umständen er sich auf die Wettkämpfe vorbereitete, interessierte einen antiken Veranstalter nicht. Preise und Privilegien waren von Anfang an wesentliche Elemente des griechischen Wettkampfwesens. Es gibt nicht den geringsten Hinweis darauf, dass materieller Gewinn einen Athleten moralisch diskriminierte, geschweige denn disqualifizierte. Während der ganzen Antike gab es neben Wettkämpfern aus wohlhabenden Familien, die Preise nicht nötig hatten, sie aber gleichwohl erstrebten oder annahmen, in zunehmendem Maße solche, die auf die genannten wirtschaftlichen Vorteile angewiesen waren. Die antiken Olympischen Spiele waren ohne Zweifel offene Spiele. 


\section{Der Primat des Sieges}

Der Kulturhistoriker Jacob Burckhardt hat die These aufgestellt, dass im Grunde alle Bereiche des Lebens der Griechen von einem agonalen Wesenszug durchdrungen gewesen seien, nicht nur die Athletik, sondern auch Politik, Wirtschaft, Kunst und Wissenschaft (Weiler, 2008). Wenngleich sein Urteil in dieser kategorischen Form vielleicht über das Ziel hinausschießt und gewisse Widersprüchlichkeiten bleiben, stimmt es in der Tendenz. Die Athletik im klassischen Griechenland war ein Modell der gnadenlosen aus der aristokratischen Standesethik heraus entstandenen Konkurrenzgesellschaft. Friedrich Schiller hat ihre entscheidenden Merkmale und Wesenszüge in einem Gedicht treffend auf den Punkt gebracht: „Von des Lebens Gütern allen ist der Ruhm das höchste doch, wenn der Leib in Staub zerfallen, lebt der große Name noch!“ (Schiller, 1962).

Ein Wettkampf hat nun einmal den Sinn, einen Sieger zu ermitteln, und wo es einen Sieger gibt, gibt es auch Unterlegene. Und hier treffen wir auf den markantesten Unterschied zwischen der Auffassung der alten Hellenen und der unsrigen: Einem antiken Athleten ging es einzig um den Sieg und um den Gewinn des damit verbundenen Preises. Nur der Sieger eines Wettbewerbs wurde von den Kampfrichtern festgestellt, offiziell ausgerufen und feierlich geehrt. Nur er hatte das Recht, im heiligen Hain von Olympia eine Statue aufzustellen, auf deren Basis sein Name, der seines Vaters und der seiner Vaterstadt stand. Nur der Sieg verschaffte einem Athleten die zunächst von den einzelnen griechischen Staaten und später vom römischen Kaiser garantierten Privilegien und materiellen Vergünstigungen. Oder, um in Anlehnung an das vielzitierte Wort des amerikanischen Trainers Vince Lombardi zu sprechen: „Winning was not the most important thing - it was the only thing!“

Sieht man einmal von den Leichenspielen in Homers Ilias ab, bei denen besondere Traditionen und kompositorische Faktoren eine Rolle spielen, so sind aus der griechischen Antike nur wenige Fälle bekannt, in denen ausnahmsweise neben den Siegern auch weitere Platzierte ermittelt und mit Preisen bedacht wurden. Nichts hätte einen Griechen wohl mehr geschockt als der Satz des modernen olympischen Credos, den der Bischof von Pennsylvania in einer Predigt in St. Paul's Cathedral bei den Olympischen Spielen 1908 in London ausgesprochen haben soll und den sich Pierre de Coubertin zu eigen machte: „Das Wichtigste bei den Spielen ist nicht zu siegen, sondern teilzunehmen" (Cook, 1909, 793).

Einem antiken Athleten ging es nämlich ausschließlich um den Sieg. Entweder man gewann und erntete Anerkennung und Beifall oder man 
unterlag und musste Ansehensverlust und Spott erfahren. Die Risikozone begann bereits mit dem zweiten Platz. Selbst die um Haaresbreite unterlegenen Finalgegner verschwanden in der namenlosen Masse, wenn sie nicht durch einen kuriosen oder dramatischen Umstand in die Anekdotensammlungen eingingen.

\section{Abbildung 7: Relief mit trainierenden antiken Fünfkämpfern}

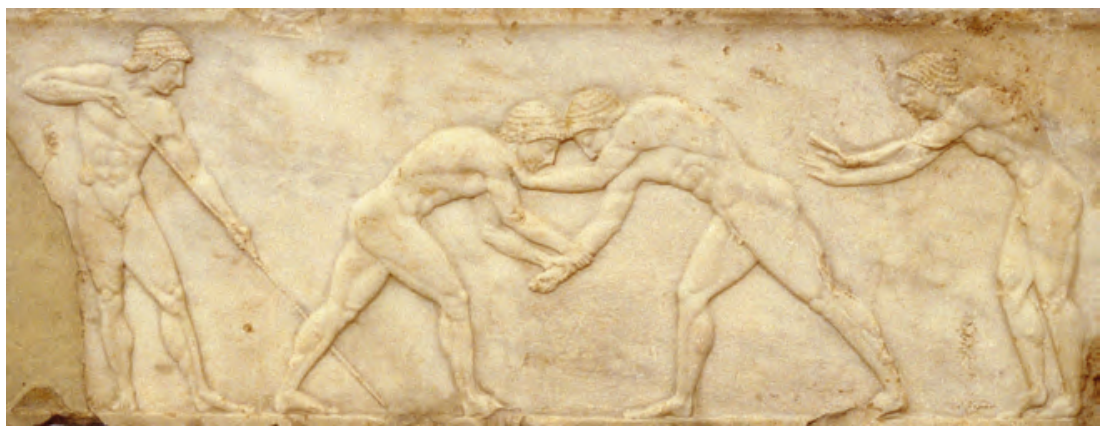

(picture alliance/Photo12/Ann Ronan Picture/Librar)

Während die Sieger unter dem Jubel ihrer Mitbürger im Triumphzug in ihre Heimatstadt einzogen und - um die Worte Pindars zu gebrauchen - „ein Leben in Honigseim“ hatten, schlichen sich die Verlierer - wie er in der 8. Pythischen Ode vermerkt - wie geprügelte Hunde heimlich und unerkannt durch Seitengassen nach Hause.

Das Bedürfnis der Griechen nach Feststellung eines Siegers trieb absurde Blüten: Wenn ein bekannter, allen anderen als überlegen angesehener Athlet bei einem Fest auftauchte, traten oft die Konkurrenten, die keine Aussicht mehr auf den Sieg zu haben glaubten, unmittelbar vor dem Wettkampf zurück, um sich für andere Gelegenheiten zu schonen, vor allem in den Kampfsportarten. Ein anderer Grund war aber auch das Bestreben, eine absehbare Niederlage und die damit verbundene Schande abzuwenden. Der übriggebliebene Champion zog allein ins Stadion ein und wurde akoniti, d. h. staublos bekränzt, was auch noch für besonders ehrenvoll gehalten wurde (Crowther, 2001). Den Griechen ging es also so sehr um die Feststellung und die Epiphanie des Siegers, dass sie sogar in besonderen Fällen den Kampf selbst als überflüssig ansahen. 
Abbildung 8: Sprunggewicht des Spartaners Akmatidas nach seinem staublosen Sieg im Fünfkampf

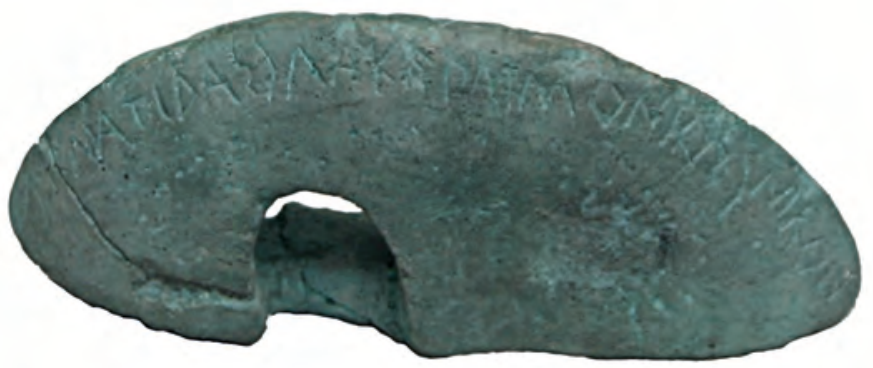

(Matthias Kabel, CC-BY-SA 3.0, via Wikimedia Commons)

Angesichts dieser Tatsachen kann es nicht verwundern, dass den Griechen ein Unentschieden nicht als ein akzeptables Ergebnis erschien. Kam es z. B. im Boxen oder Pankration selbst nach stundenlangem Kampf zu keiner Entscheidung, wurden nicht, wie wir erwarten würden, beide Athleten zu Siegern erklärt, sondern keiner. Der Kranz wurde dem Gott geweiht, dem der Wettkampf galt. Erst in der zu Unrecht verketzerten römischen Kaiserzeit änderte sich diese starre Auffassung ein wenig. Es kam vereinzelt zu Kampfpreisteilungen bei unentschiedenem Ausgang von Wettbewerben und zur Errichtung von Statuen für Athleten, die zwar nicht gesiegt, aber mit Bravour gekämpft hatten.

Bei den Panhellenischen Spielen wurde mit äußerster Kompromisslosigkeit gekämpft. Selbst das Leben wurde eingesetzt, um die Schande einer Niederlage abzuwenden: „Kranz oder Tod“ heißt die rigorose Devise, die auf dem Grabstein eines Athleten aus Alexandria in Olympia gefunden wurde (Finley \& Pleket, 1976, S. 190). Spricht diese Devise nicht jeder Humanität Hohn?

Nicht selten kam es zu neurotischen Verhaltensweisen und Selbstmorden von Unterlegenen. Der spartanische Ehrenkodex schrieb vor, lieber den Tod auf sich zu nehmen, als sich in der Schlacht oder im Agon zu ergeben. Dies hatte zur Folge, dass Sparta von einem gewissen Zeitpunkt an das Pankration, eine dem Ringen verwandte Kampfsportdisziplin, die nur dann entschieden war, wenn sich einer der Kontrahenten ausdrücklich für besiegt erklärte, nicht mehr beschickte (Poliakoff, S. 80/81).

Der Grieche dachte in der Athletik ausschließlich in Kategorien des persönlichen Vergleichs: Er wollte wissen, wer der beste 
Einzelkämpfer war. Kennzeichnend für diese Denkweise war das für uns schwer nachzuvollziehende System der Siegerermittlung im Fünfkampf (Diskuswurf, Sprung, Speerwurf, Lauf, Ringen). Man kannte keine Punktwertung wie heute, sondern löste den Wettkampf gedanklich so auf, dass man das Abschneiden jedes einzelnen Athleten mit jedem einzelnen verglich und jeden, der dreimal gegen ein und denselben Konkurrenten verloren hatte, ausscheiden ließ (Ebert, 1963). Somit waren viele antike Fünfkämpfer, die nicht der obersten Leistungsklasse angehörten, eigentlich zeitlebens Drei- oder Vierkämpfer. Die Ermittlung des Siegers stand also so im Vordergrund, dass man jedem Athleten die weitere Teilnahme verwehrte, den man nicht mehr für die Entscheidung um den ersten Platz benötigte oder der selbst keine Aussicht mehr auf den Sieg hatte. Angesichts der bisher dargestellten Fakten verwundert es nicht, dass es in der Antike keine Mannschaftswettbewerbe gab. Die Selbstdarstellung des Individuums stand im Vordergrund. Angesichts dieser Unbedingtheit und Kompromisslosigkeit kann man die immer wieder zu lesenden Forderungen in den Feuilleton-Spalten der Sportpresse, die Olympischen Spiele, die in einen so erbarmungslosen Existenzkampf ausgeartet seien, müssten wieder „wirkliche Spiele“ wie in der Antike werden, nur als Illusion bezeichnen. Die antike Athletik hatte einen so ernsthaften Charakter und wies, vor allem in den Kampfsportarten, ein solches Ausmaß an Aggression und Gewalt auf, dass wir sie eher dem Krieg als dem Sport zuweisen würden. Von Spielen kann keine Rede sein. Das Wort agon bedeutete im Griechischen Kampf, und zwar gleichermaßen im Sinne von Krieg/Schlacht und sportlichem Wettkampf. Der griechische Historiker Thukydides bezeichnete den 30-jährigen Peloponnesischen Krieg zwischen Athen und Sparta als agon. Auch heute heißen die Olympischen Spiele, abweichend von der Bezeichnung in anderen Sprachen, im Neugriechischen agones olympikoi, also Olympische Kämpfe.

\section{Leistungsbegriff und Leistungsmessung in der antiken Athletik}

Unsere heutige Vorstellung von der sportlichen Leistung ist in der Regel eng gebunden an quantifizierbare Kategorien, d. h. an eine exakte Messung von Zeiten, Weiten, Höhen, Gewichten oder ein möglichst objektives System von Punktwertungen oder Zählungen.

Unser Leistungsbegriff hat sich in der Zeit der Aufklärung herausgebildet und in engem Zusammenhang mit der Industrialisierung und dem technischen Fortschritt im 19. Jahrhundert weiterentwickelt (Eichberg, 1979; Grupe, 1982). Der von dem französischen Jesuitenpater Didon 
formulierte und von Pierre de Coubertin propagierte Wahlspruch „Citius - Altius - Fortius" verrät deutlich, dass sich die ihm zugrundeliegende Vorstellung vom Sport vorwiegend an der Messung in Zentimeter, Gramm und Sekunden orientierte. Nicht nur der Sieg, sondern auch der absolute Rekord gehörten nach Coubertin zu den wesentlichen Sinnelementen des Olympismus. Im 20. Jahrhundert hat man durch Normierung und Optimierung von Wettkampfstätten, Sportkleidung und Sportgeräten sowie durch die wissenschaftliche Erforschung der Trainingswirkungen in den meisten Sportarten die absoluten Rekorde ständig verbessert, bis man in den letzten Jahren an Leistungsgrenzen gestoßen zu sein scheint, die nur noch durch massive Manipulation der natürlichen Ressourcen des menschlichen Organismus weiter herausgeschoben werden können.

\section{Abbildung 9: Antike Langläufer}

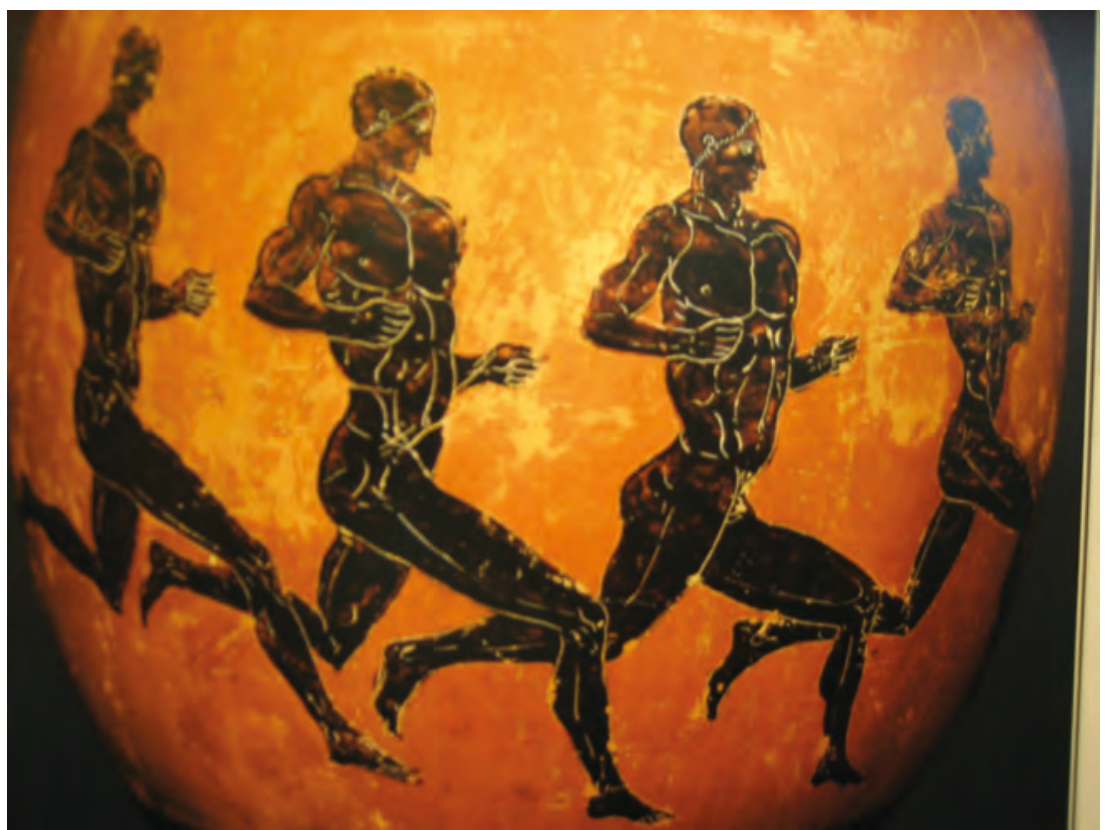

(Archiv Manfred Lämmer)

Mit derartigen Problemen hatten die Griechen nichts zu tun. Bei ihren Wettkämpfen wurden weder Zeiten oder Weiten gemessen noch Punktetabellen benutzt. Rekorde in unserem Sinne konnten schon deshalb nicht aufgestellt werden, weil Sportstätten und Geräte nicht standardi- 
siert waren. Die ca. 300 griechischen Stadtstaaten wachten eifersüchtig über ihre Autonomie, gaben sich ihre eigenen Gesetze und benutzten eigene Kalender, Maße und Gewichte. Diese mussten im Verkehr mit den Nachbarn im Bedarfsfalle mit Hilfe von Konkordanzlisten umgerechnet werden.

\section{Abbildung 10: Eine dynamische Szene mit Sprintern}

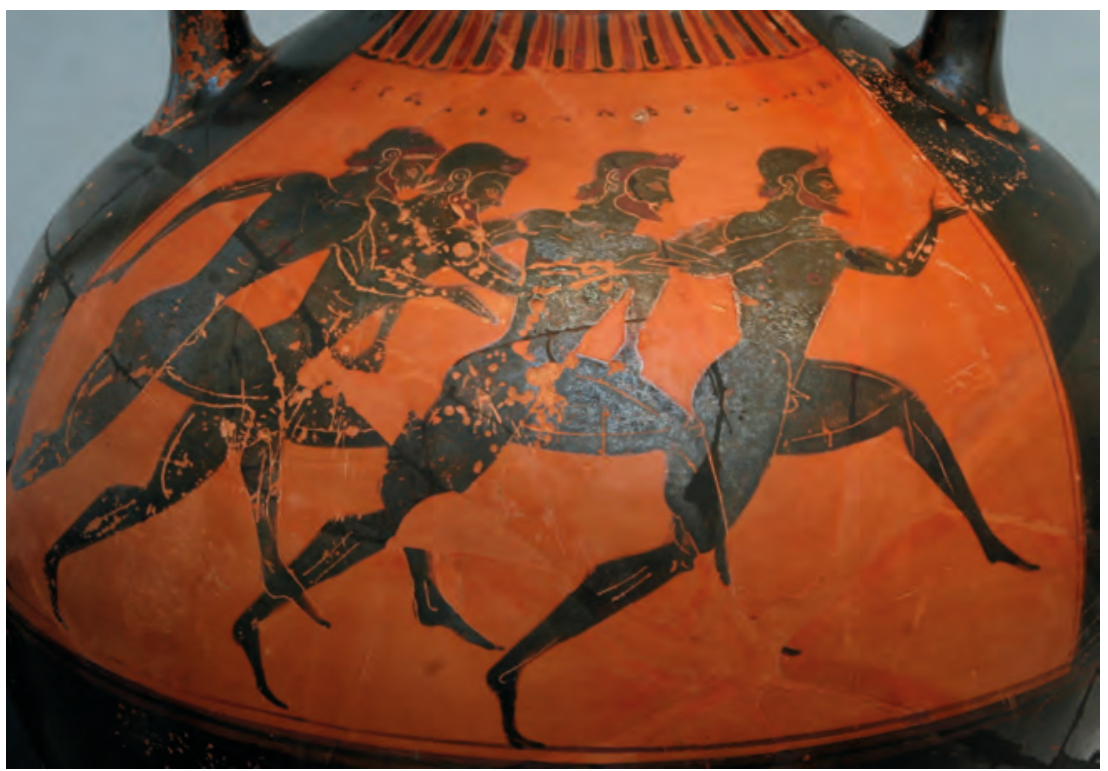

(Matthias Kabel, CC-BY-SA 3.0, via Wikimedia Commons)

Ein Beispiel dafür, das sich auf die Leichtathletik bezieht: Das Wort Stadion ist ein altgriechisches Längenmaß, mit dem in der Antike allgemein räumliche Distanzen angegeben wurden, wie wir das heute mit den Begriffen Kilometer oder Meilen tun. Ein Stadion zählte in allen griechischen Städten gleichermaßen 600 Fuß, doch da die Länge des Fußes lokal verschieden war, ergaben sich für die Laufbahnen der Stadien, in denen die athletischen Wettkämpfe stattfanden, große Differenzen. Die Bahn in Olympia, die Herakles selbst abgemessen haben soll, hatte eine Länge von 192,28 m, die in Athen 184 m, die in Delphi 178 m, die in Delos nur 168 $\mathrm{m}$, die in Pergamon dagegen $210 \mathrm{~m}$. Schon daraus wird ersichtlich, dass es keine normierten Laufstrecken gab, die - selbst wenn man es technisch vermocht hätte - Grundlage einer einheitlichen Zeitmessung und der Aufstellung von Rekordlisten gewesen wäre. 
Das gleiche trifft für die Sportgeräte zu. Bis heute hat man nicht zwei hinsichtlich ihres Durchmessers und ihres Gewichtes identische Disken gefunden. Selbstverständlich waren die Sportgeräte, die bei ein und demselben Wettkampf zum Einsatz kamen, gleich. Pausanias berichtet, dass die Fünfkämpfer in Olympia drei Disken gleicher Größe benutzten, die zwischen den Spielen im Schatzhaus der Stadt Sikyon aufbewahrt wurden (Pausanias VI, 19,4). Im Zeus-Tempel hingen 25 typengleiche Schilde, die beim Waffenlauf Verwendung fanden (Pausanias V, 12,8). Wir dürfen davon ausgehen, dass ähnliche Vorkehrungen für den Speerwurf und den Weitsprung getroffen wurden. So waren die Voraussetzungen für alle Athleten gleich. Die erzielten Leistungen wurden allerdings nicht gemessen, sondern der Aufschlagpunkt bzw. der letzte Abdruck des Fußes beim Weitsprung durch einen Pflock gekennzeichnet. Da Diskuswurf, Speerwurf und Weitsprung nur im Fünfkampf ausgetragen wurden, ging es ohnehin nur um die Feststellung der Platzierung, um mit Hilfe des bereits angesprochenen Prinzips den Sieger zu ermitteln.

In der 1.000-jährigen Geschichte der antiken Athletik verzeichnen wir nur wenige Abweichungen von diesen Gepflogenheiten. Der Grieche war offensichtlich nicht in der Lage, seine Vorstellung von einer athletischen Leistung an abstrakten, numerischen Kategorien zu orientieren: Bekannt ist die von einem römischen Schriftsteller überlieferte Anekdote: Ein Athlet, der bei einem Wettkampf eine schlechte Form aufwies, versuchte diese Tatsache dadurch zu kompensieren, dass er darauf hinwies, bei einem Wettkampf auf Rhodos eine Superweite erreicht zu haben. Sein Mitkonkurrent wischte die Antwort vom Tisch: „Hic Rhodus - hic salta!“ - „Hier ist Rhodos - spring hier!“ (Aisopos, 203b).

Während heute ein Athlet die Möglichkeit hat, innerhalb eines gewissen Zeitraums eine geforderte Qualifikation zu erreichen, ohne mit einem Konkurrenten direkt aufeinander getroffen zu sein, war dies in der Antike nicht möglich. Leistungen mussten immer zu einem ganz konkreten Zeitpunkt erbracht werden.

Die griechische Sitte, keine messbaren Leistungen aufzuzeichnen, hatte noch eine andere wichtige Folge. Ein Vergleich von Ergebnissen, die in verschiedenen Epochen erbracht wurden, war nicht möglich. Obwohl gar kein Zweifel daran bestehen kann, dass die professionalisierten und intensiv betreuten Athleten der römischen Kaiserzeit weitaus besser waren als die der klassischen Epoche, blieb der Ruhm der Alten für immer erhalten. In der Kaiserzeit wird der angebliche Verfall der Athletik beklagt und bedauert, dass es keine überragenden Persönlichkeiten mehr gebe wie die des Ringers Milon, der sechs Mal in Folge (540-516 v.Chr.) bei den Olympischen Spielen den Kranz errungen habe (Philostratos, 2014, 
Gymnastikos 1. Poliakoff, 1989). Diese Tatsache signalisiert uns heute genau das Gegenteil, dass nämlich die Konkurrenz in der vielgepriesenen alten Zeit offensichtlich so schwach war, dass Milon so lange unangefochten dominieren konnte.

Aus dem bisher Gesagten ergibt sich noch eine interessante praktische Konsequenz für die Durchführung der großen Wettkämpfe, insbesondere für die Olympischen Spiele. Da keine Leistungen gemessen und öffentlich kommuniziert wurden, die Athleten aber nur selten mit ihren Konkurrenten in einen direkten Wettkampf traten, konnten sie ihr eigenes Leistungsniveau kaum verlässlich einschätzen. Nun konnte sich aber soweit wir dies aus den Quellen entnehmen - jeder freie Grieche, der Geld, Zeit und Selbstvertrauen hatte, zur Teilnahme an den Olympischen Spielen melden. Dass aber schließlich immer nur eine Handvoll Top-Stars in Olympia antraten, ist eine Folge der Bestimmung, dass alle Teilnahmewilligen sich einen Monat vor Beginn der Spiele in Elis, der $40 \mathrm{~km}$ von Olympia entfernt gelegenen Veranstalterstadt, zu melden hatten. Die obersten Schiedsrichter, die Hellanodiken, beobachteten die Bewerber vier Wochen lang beim Training und wiesen alle, die sich offenbar überschätzt und keine Aussicht auf den Sieg hatten, ab (Ortkemper, 1996). Heute übernehmen diese Aufgabe die Nationalen Olympischen Komitees auf Grund von festgelegten Normen der Internationalen Sportfachverbände, die sich während eines bestimmten Zeitraums ein genaues Bild vom Leistungsniveau eines jeden Athleten gemacht haben.

Die Leistung eines griechischen Athleten bestand nicht in einem messbaren Ergebnis, sondern in der Überwindung des Gegners. Der Ringkampf war zu Ende, wenn der Gegner drei Gänge verloren hatte, Boxkampf und Pankration kannten keine Punktwertung, sondern wurden ausschließlich durch Kampfunfähigkeit eines der Kontrahenten oder ausdrückliche Aufgabe entschieden. Dem "Citius - Altius - Fortius“ Coubertins hält der Grieche den oben erwähnten Leitspruch „Immer der erste sein und überlegen den anderen" entgegen. 


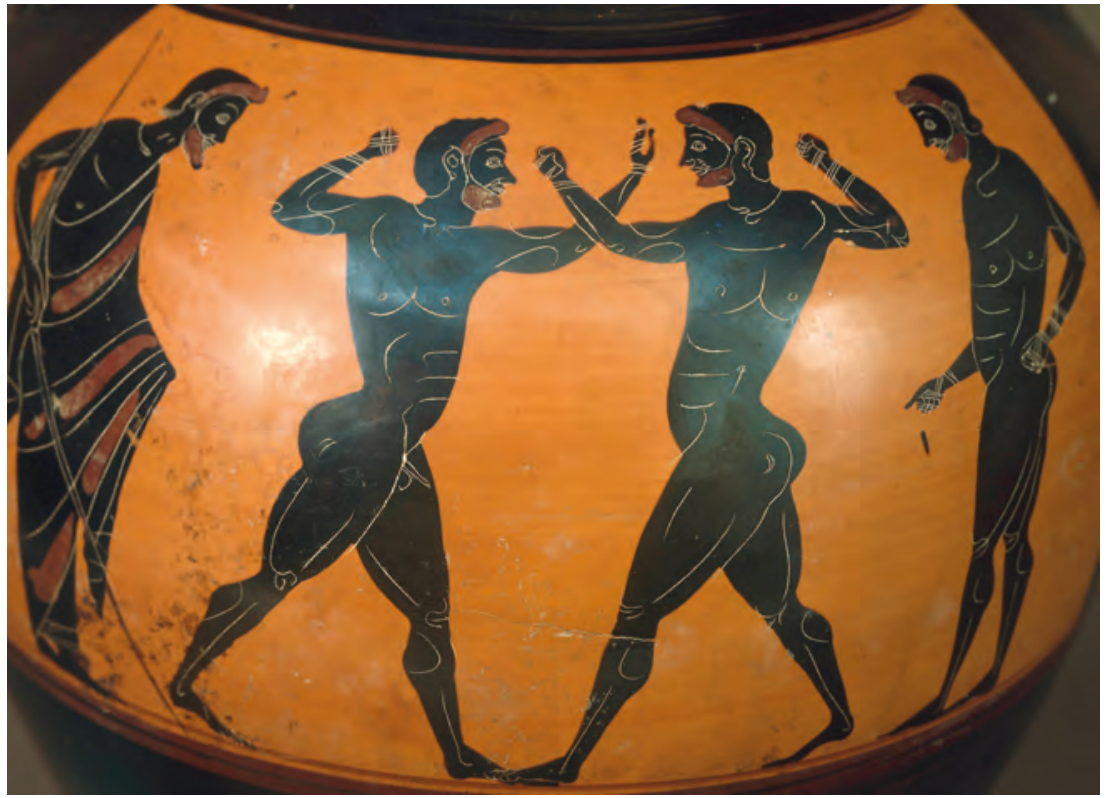

(picture alliance/akg-images/Nimatallah)

Obwohl die Griechen keine Rekordlisten in unserem Sinn führten, versuchten sie dennoch, einer als hervorragend empfundenen Leistung über die Feststellung des Sieges hinaus auf andere Weise den Charakter der Einmaligkeit bzw. besonderen Denkwürdigkeit zu verleihen: Da ihnen die Zahl nicht zur Verfügung stand, bildete sich die Sitte heraus, außergewöhnliche Kombinationen von Siegen, besondere Erfolgsserien oder staunenswerte Umstände ihrer Kampfesweise durch spezielle Attribute zu kennzeichnen, wodurch eine Reihe von begehrten Ehrentiteln entstand. Der bedeutendste war der des Periodoniken (Knab, 1934). Er wurde dem Athleten verliehen, der bei allen vier Panhellenischen Spielen, den Olympien, Pythien, Isthmien und Nemeen, in seiner Disziplin siegte. Dieser Zyklus wurde nämlich Periodos, d.h. Umlauf genannt. Der Titel ist mit dem des Grand Slam im modernen Tennis vergleichbar. Den Grand Slam hat bekanntlich ein Spieler bzw. eine Spielerin gewonnen, dem/der es gelungen ist, innerhalb einer Saison bei den vier bedeutendsten Turnieren der Welt (Australian Open, Paris Open, Wimbledon und U. S. Open) zu siegen. 
Es fällt auf, dass in einer Gesellschaft, die so sehr das Konkurrenzprinzip kultivierte, das Gefühl für Chancengleichheit relativ gering ausgeprägt war. Nur wenige archetypische Regeln sicherten den Verlauf der Wettkämpfe. Ihre Einhaltung musste zudem durch den Olympischen Eid gesichert werden (Lämmer, 1995). Die organisatorischen Gepflogenheiten waren nicht ohne Widersprüche: Einerseits kannte man eine Einteilung der Wettkämpfer in zwei (so in Olympia) oder drei Alterskategorien, so dass die sich noch in der körperlichen Entwicklung befindlichen Jugendlichen unter sich blieben, andererseits gab es in den Kampfsportarten Ringen, Pankration und Boxen keine Gewichtsklassen (Ebert, 1980, S. 63/64). Im Übrigen interessierte es niemanden, wie sich ein Athlet auf die Wettkämpfe vorbereitete und in welchen sozialen Verhältnissen er sich befand. Dies war allenfalls Sache der Heimatstädte. Man empfand es auch nicht als Ungerechtigkeit und Benachteiligung, dass Frauen und Nichtgriechen von der Teilnahme an Wettkämpfen ausgeschlossen waren entsprach dies doch ihrer allgemeinen Ausgrenzung aus dem politischen Leben.

Ein Phänomen ist aber noch von besonderem Interesse: Die Griechen waren zutiefst davon überzeugt, dass der Erfolg im Wettkampf nur zum Teil Ergebnis eigenen Bemühens und eigener Leistung war. Letztlich entschieden über Sieg und Niederlage die Götter, die oft willkürlich helfend oder behindernd in den Wettkampf eingriffen. Dies wird in hervorragender Weise an der Schilderung der Wettkämpfe zu Ehren des Patroklos im 23. Gesang der Ilias deutlich (Ilias, 23, 257-897). Fast alle Wettbewerbe werden durch die Götter entschieden, während die Athleten versuchen, sich durch Gebet und Opfer ihrer Hilfe zu versichern. Letztlich wird hier der Mensch zum Spielball höherer Mächte, was allerdings den Vorteil hat, dass er eine Niederlage nicht auf eigene mangelnde Leistungsfähigkeit zurückführen muss, sondern sie auf Kräfte und Einflüsse schieben kann, die sich seiner Einwirkungsmöglichkeit entziehen.

Pausanias berichtet, dass die Olympiakämpfer, die sich monatelang intensiv vorbereitet hatten und von den Hellanodiken sorgfältig überprüft worden waren, vor dem Betreten des Stadions an einem besonderen Altar dem Kairos, dem „Gott des günstigen Augenblicks“, opferten - wir würden sagen: dem „Gott der Tagesform“ (Pausanias V 14, 9). 


\section{Fazit}

Pierre de Coubertin hat das Prinzip, von dem er sich bei der Wiederbelebung der Olympischen Spiele leiten ließ, mit der Formel umschrieben: „Antiken Sinn und moderne Form vereinen“ (Cook, 1909, S. 197). Diesen Anspruch können die modernen Spiele nicht erfüllen. Das antike Amateurideal ist eine fromme Lüge in wohlgemeinter pädagogischer Absicht, die friedensstiftende Mission der Hellenischen Spiele eine Illusion unserer Zeit, der unbedingte Vorrang des Sieges und der verbissene Ernst, den viele am heutigen Wettkampfgeschehen so sehr bedauern, waren dagegen typisch griechisch. Nationale und soziale Restriktion machten die antiken Spiele zur Angelegenheit einer privilegierten Minderheit. Die Aufzählung weiterer Unterschiede ließe sich fortführen. Es fällt schwer, in der Antike überhaupt einen einzigen Kernbegriff des modernen Olympismus wiederzuentdecken. Die Spiele der Antike unterschieden sich von denen, die Coubertin gründete, so grundlegend von jenen, die wir heute erleben.

Abbildung 12: Das antike Olympiastadion - eine überraschend schlichte Anlage

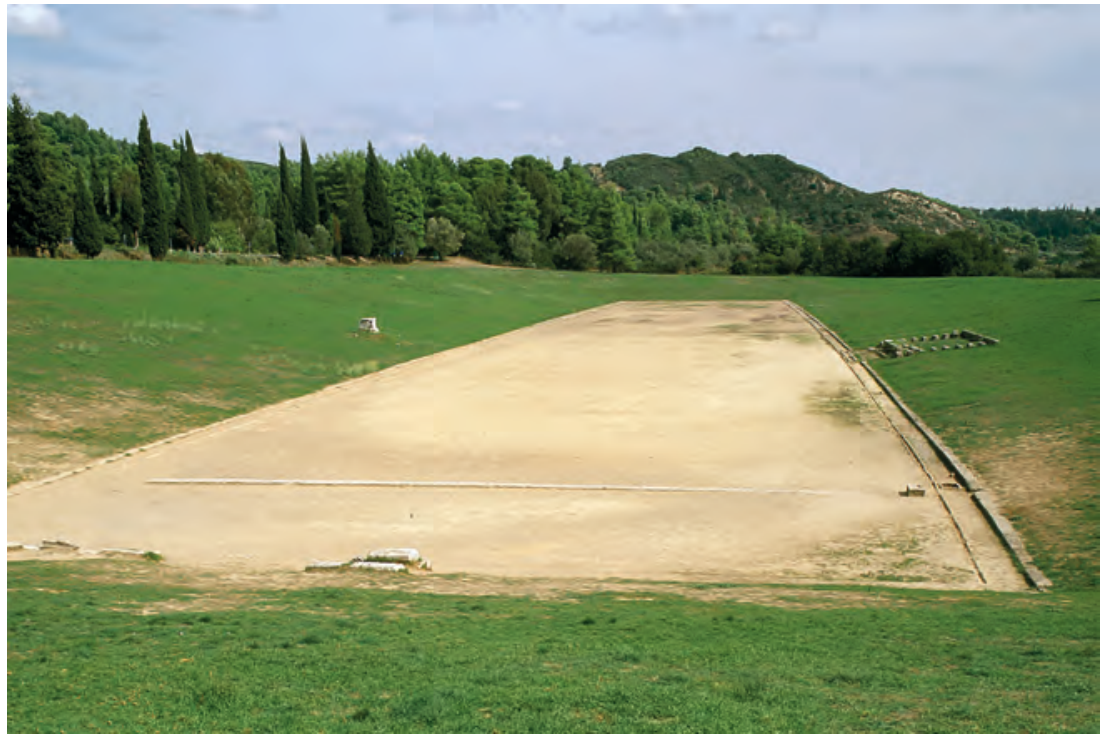

(picture-alliance/Herve Champollion/akg-images) 
Abbildung 13: Das Stadion der ersten Olympischen Spiele der Neuzeit 1896 in Athen

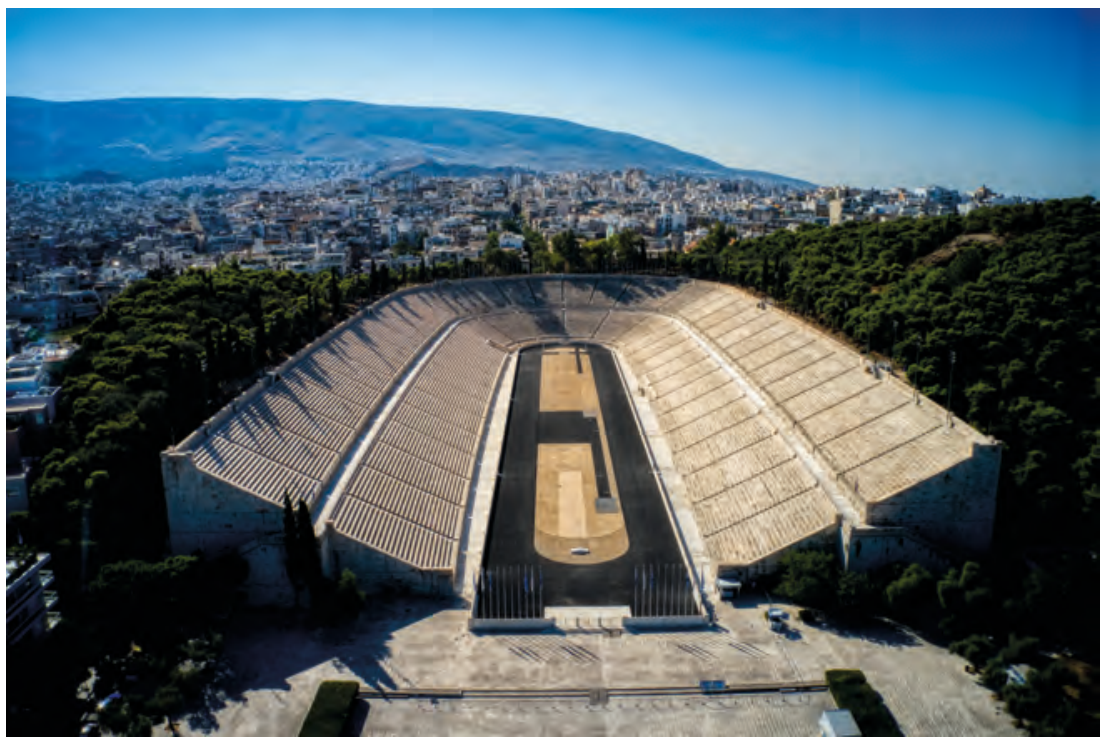

(picture alliance/ANE/Eurokinissi/Antonis Nikolopoulos)

In der Andersartigkeit unserer Wertvorstellungen und Maßstäbe manifestiert sich eben der große geistige, kulturelle und gesellschaftliche Fortschritt seit der Antike, den wir dem Christentum, der Reformation, der Aufklärung und den modernen sozialen Bewegungen verdanken. Die kurze Rückschau hat gezeigt, dass sich die Olympische Idee letztlich reduzieren lässt auf das Prinzip periodisch wiederkehrender festlich gestalteter Wettkämpfe, deren Sinngebung sich wandelt und immer wieder neu gefunden werden muss.

Doch ein Paradoxon bleibt: Das weitverbreitete falsche Bild von den antiken Olympischen Spielen war eine der wirksamsten Triebkräfte für die Entwicklung und den Erfolg der Coubertinschen Idee. 


\section{Literatur:}

Cook, Th. (1909). The Fourth Olympiad being The Official Report. The Olympic Games of 1908 celebrated in London. London: The British Olympic Association.

Coubertin, P. de (1966). Die philosophischen Grundlagen des modernen Olympismus. In Carl-Diem-Institut (Hrsg.), Pierre de Coubertin. Der Olympische Gedanke. Reden und Aufsätze. Schorndorf: Karl Hofmann.

Crowther, N.B. (2001). Victories without Competition in the Greek Games. Nikephoros, 14, 29-44.

Decker, W. (2012). Sport in der griechischen Antike. Vom minoischen Wettkampf bis zu den Olympischen Spielen (2. Aufl.). München: Verlag C.H. Beck.

Ebert, J. (1963). Zum Pentathlon der Antike. Untersuchungen über das System der Siegerermittlung und die Ausfübrung des Halterensprungs (Abhandlungen der Sächsischen Akademie der Wissenschaften zu Leipzig, phil.-hist., Klasse 56,1). Berlin [DDR]: Akademie-Verlag.

Ebert, J. (1980). Olympia von den Anfängen bis zu Coubertin. Leipzig: Koehler \& Amelang.

Eichberg, H. (1979). Der Weg des Sports in die industrielle Zivilisation. Baden-Baden: Nomos.

Finley, M. I. \& Pleket, H. W. (1976). Die Olympischen Spiele der Antike. Tübingen: R. Wunderlich Verlag.

Fricke, K. (1982). Die Idee der ständigen Austragung der Olympischen Spiele in Griechenland. Diplomarbeit, Deutsche Sporthochschule Köln.

Grupe, O. (1982). Bewegung, Spiel und Leistung im Sport. Grundthemen der Sportanthropologie. Schorndorf: Karl Hofmann.

Homer (2013). Ilias. Übersetzt von H. Rupé. Berlin: De Gruyter.

Höfer, A. (1994). Der Olympische Friede. Anspruch und Wirklichkeit einer Idee (Studien zur Sportgeschichte, Band 2). St. Augustin: Academia.

Knab, R. (1934). Die Periodoniken. Ein Beitrag zur Geschichte der gymnischen Agone an den vier griechischen Hauptfesten. Dissertation, Gießen.

Lämmer, M. (1980). Flucht nach Hellas? Griechenlands Vorschlag. Olympisches Feuer, 30(4), 43-47.

Lämmer, M. (1981). Women and Sport in Ancient Greece. A Plea for a Critical and Objective Approach. In J. Borms (Hrsg.), Women and Sport. An Historical, Biological, Physiological and Sportsmedical Approach (S. 16-23). International Congress, Rome, July 1980.

Lämmer, M. (1982). Der sogenannte Olympische Friede in der griechischen Antike. Stadion, 8/9, 47-83.

Lämmer, M. (1985) Zum Verhältnis von Sport und Krieg in der griechischen Antike. In H. Becker (Hrsg.), Sport im Spannungsfeld von Krieg und Frieden (S. 17-30). Clausthal-Zellerfeld: Deutsche Vereinigung für Sportwissenschaft. 
Lämmer, M. (1995). Der Olympische Eid in der griechischen Antike. In H. Schaller \& D. Pache (Hrsg.), Sport als Bildungschance und Lebensform. Prof. Dr. Heinz Denk zum 65. Geburtstag gewidmet (S. 157-164). Schorndorf: Karl Hofmann.

Olympische Charta (2014). Übersetzt von C. Vedder \& M. Lämmer im Auftrage der Deutschen Olympischen Akademie. Melsungen: Bernecker.

Ortkemper, H. (1996). Olympische Legenden. Geschichten aus dem antiken Olympia. Insel Verlag.

Pausanias (1954). Beschreibung Griechenlands, neu übersetzt von E. Meyer. Zürich: Artemis-Verlag.

Philostratus (2014). Heroicus. Gymnasticus. Discourses 1 and 2 (Edited and Translated by Jeffrey Rusten and Jason König). Cambridge, Mass./London, England: Harvard University Press.

Poliakoff, M. B. (1989). Kampfsport in der Antike. Das Spiel um Leben und Tod. München: Artemis \& Winkler.

Schiller, F. (1962). Das Siegesfest [1803]. Sämtliche Werke, Band 1, 382-383. München: Hanser.

Sinn, U. (2004). Das antike Olympia. Götter, Spiel und Kunst. München: Verlag C.H. Beck.

Swaddling, J. (2004). Die Olympischen Spiele der Antike. Stuttgart: Reclam.

Weeber, K.-W. (1991). Die unheiligen Spiele. Das antike Olympia zwischen Legende und Wirklichkeit. Artemis \& Winkler.

Weiler, I. (1975). AIEN APICTEYEIN. Ideologiekritische Bemerkungen zu einem vielzitierten Homerwort. Stadion, 1(1), 199-227.

Weiler, I. (2008). Der Agon, die Agonalität und das Agonale aus der Sicht des Althistorikers. Leipziger sportwissenschaftliche Beiträge, 49(1), 4-26.

Young, D.C. (1984). The Olympic Myth of Greek Amateur Athletics. Chicago: Ares. 
